\title{
Criminologie
}

\section{Un paradigme développemental pour la criminologie : développement et autorégulation de la conduite déviante}

\section{Marc Le Blanc}

Volume 43, numéro 2, automne 2010

Les 50 ans de l'École de criminologie : aperçu de la recherche d'ici et d'ailleurs

URI : https://id.erudit.org/iderudit/1001783ar

DOI : https://doi.org/10.7202/1001783ar

Aller au sommaire du numéro

Éditeur(s)

Les Presses de l’Université de Montréal

ISSN

0316-0041 (imprimé)

1492-1367 (numérique)

Découvrir la revue

Citer cet article

Le Blanc, M. (2010). Un paradigme développemental pour la criminologie :

développement et autorégulation de la conduite déviante. Criminologie, 43(2), 401-428. https://doi.org/10.7202/1001783ar
Résumé de l'article

Le présent article résume les progrès réalisés par la criminologie en présentant les origines et les étapes de la construction du paradigme développemental pour la compréhension de la conduite déviante des personnes. Après avoir défini les objets de ce paradigme, cet article décrit la structure hiérarchique du syndrome de la conduite déviante. Il précise les paramètres qui permettent de décrire chacune des formes de ce syndrome dans son état actuel et les changements qu'elles subissent. Les mécanismes de sa construction (l'activation, l'aggravation et le désistement) et les trajectoires que prennent les formes de la conduite déviante sont exposées. Cette présentation du paradigme développemental est complétée par un tableau de son autorégulation et par une indication de son application à la compréhension du fonctionnement des causes des formes de la conduite déviante. 


\title{
Un paradigme développemental pour la criminologie: développement et autorégulation de la conduite déviante
}

\author{
Marc Le Blanc \\ Professeur émérite \\ École de criminologie et École de psychoéducation, Université de Montréal \\ Docteur honoris causa en criminologie et psychologie, Université de Liège \\ leblancm@psyced.umontreal.ca
}

RÉSUMÉ • Le présent article résume les progrès réalisés par la criminologie en présentant les origines et les étapes de la construction du paradigme développemental pour la compréhension de la conduite déviante des personnes. Après avoir défini les objets de ce paradigme, cet article décrit la structure hiérarchique du syndrome de la conduite déviante. Il précise les paramètres qui permettent de décrire chacune des formes de ce syndrome dans son état actuel et les changements qu'elles subissent. Les mécanismes de sa construction (l'activation, l'aggravation et le désistement) et les trajectoires que prennent les formes de la conduite déviante sont exposées. Cette présentation du paradigme développemental est complétée par un tableau de son autorégulation et par une indication de son application à la compréhension du fonctionnement des causes des formes de la conduite déviante.

MOTS-CLÉS - Conduite déviante, développement, changements quantitatifs et qualitatifs, mécanismes d'activation, d'aggravation et de désistement, autorégulation.

\section{Introduction $^{1}$}

Le paradigme développemental propose d'étudier les changements qui s'opèrent dans la conduite déviante des personnes tout au long de leur

1. Les activités de recherche sous-jacentes à cet article ont été subventionnées pendant de nombreuses années par le Conseil de recherches en sciences humaines du Canada, le Conseil québécois de la recherche sociale, la Bourse Killam et le Fonds pour la Formation de chercheurs et l'aide à la recherche du Québec. 
vie et de reconnaître les facteurs qui expliquent son apparition, son développement et son extinction. Il a été formalisé dans l'étude empirique de Le Blanc et Fréchette (1989) et dans la recension des écrits de Le Blanc et Loeber (1998; voir aussi Loeber et Le Blanc, 1990). Le paradigme développemental s'appuie sur une longue tradition en criminologie.

Il a été anticipé par Quételet (1842) à la faveur de ses travaux sur les rapports entre l'âge et l'activité criminelle. Il a trouvé sa méthode empirique grâce à l'exemple des études longitudinales des Glueck (1930, 1937, 1940, 1943, 1958). Ce paradigme a commencé à prendre forme, dès les années 1970, dans les travaux dirigés par Jacques Selosse à Vaucresson (Breuvart et al., 1974). Ces chercheurs utilisent la notion de «carrière criminelle» pour englober des notions comme la récidive, le genre de vie délinquant, la conduite délinquante et les types de criminels. Ils spécifient que l'étude de la carrière criminelle doit s'appuyer sur l'analyse des rapports entre quatre paramètres: la précocité, le polymorphisme, la fréquence des délits et la gravité des peines. Cette étude de données sur la délinquance officielle montre, pour la première fois en criminologie, comment le polymorphisme est associé à la précocité, à la gravité et à la fréquence des délits.

Il aura fallu attendre le milieu des années 1980, avec Blumstein et al. (1986), pour que la criminologie fasse de la carrière criminelle un de ses objets centraux de recherche et de théorisation. Blumstein et ses collaborateurs (1986) concevaient la carrière criminelle comme étant composée d'abord de paramètres descriptifs : la participation à la délinquance, la fréquence des infractions et leur gravité. Ils ajoutaient des paramètres de délimitation: l'âge du début, la durée et l'âge d'arrêt de la carrière criminelle. Depuis, une quantité impressionnante de travaux est venue enrichir la compréhension de ces phénomènes, l'analyse des liens entre eux et la recherche de facteurs qui rendent compte de l'initiation, du maintien et de l'arrêt de l'activité criminelle. La recension récente de ces travaux par Piquero et al. (2003) fait référence à environ 250 publications au cours des vingt-cinq dernières années.

Le paradigme développemental intègre la perspective de la carrière criminelle parce que les mêmes paramètres descriptifs et de délimitation sont employés. Par contre, il la dépasse par plusieurs caractéristiques. Il ne se limite pas aux seules activités criminelles, mais il considère l'ensemble des formes de la conduite déviante et des interactions entre elles à travers le temps. Si le paradigme développemental se sert des 
mêmes descripteurs de l'activité criminelle, il ajoute l'existence de mécanismes du cycle des activités déviantes dans la vie des personnes. La perspective de la carrière criminelle s'appuie principalement sur des études empiriques avec les données de la délinquance officielle. Pour sa part, le paradigme développemental considère ces données, mais il privilégie les données qui proviennent de l'activité déviante rapportée par les personnes et, surtout, il s'appuie sur des échantillons représentatifs de la population.

Le paradigme développemental se distingue aussi par la stratégie de recherche choisie, les études longitudinales. La plupart des travaux étiologiques en criminologie sont, depuis toujours, des études transversales. Elles permettent de reconnaitre les facteurs qui sont statistiquement associés à la conduite déviante et les interactions entre eux à un moment donné dans la vie des personnes. Par contre, elles ne comparent pas et elles n'ordonnent pas les facteurs tout au long de la vie des personnes. Elles ne distinguent pas les facteurs d'apparition, de maintien et d'extinction de la conduite déviante. Le paradigme développemental cherche à distinguer les causes parmi les facteurs explicatifs de la conduite délinquante tout au long de la vie des personnes. Il rejoint un paradigme central en psychologie, le développement de la personne (Lerner, 2002), et en sociologie, le cycle de la vie sociale (Elder, 1992); ces derniers n'avaient pas été introduits en criminologie.

Ce paradigme propose donc deux champs spécifiques d'étude. Premièrement, il s'agit d'analyser la dynamique de la conduite déviante à partir de l'enfance jusqu'à la fin de la vie. Deuxièmement, il s'agit de distinguer les facteurs explicatifs qui précèdent, qui accompagnent ou qui suivent les changements dans la conduite déviante à travers le temps, c'est-à-dire la séquence des causes et des conséquences biologiques, psychologiques et sociales qui affectent la conduite déviante des personnes. Cet article porte sur le premier champ d'étude.

\section{La conduite déviante, un syndrome hétérotypique et cyclique}

La criminologie développementale doit rendre compte des transformations de la conformité aux standards conventionnels de la conduite au cours de la vie des personnes. Cette notion de la conformité aux standards de la conduite constitue la position classique en criminologie (Hirschi, 1969; Fréchette et Le Blanc, 1987). Elle se rapporte à la norme 
pour les comportements dans une société, à un moment donné et pour un groupe d'âge. La diversité des comportements regroupés sous le terme de «conduite déviante» s'allonge sur un continuum allant des activités jugées par les adultes comme impropres à un mineur (les relations sexuelles, l'usage de l'alcool et des substances psychoactives illicites, l'opposition aux parents et le conflit avec les autorités, etc.) jusqu'aux délits que le Code criminel définit avec précision (homicide, assaut, vol à main armée, vol d'une automobile, vol avec effraction, etc.). Les comportements prohibés par les lois et les règlements édictés spécialement pour les adolescents sont inclus dans cette appellation de la conduite déviante (la conduite automobile inappropriée, la non-fréquentation scolaire, la présence dans les débits de boisson, les troubles graves du comportement, etc.). Le terme de «délinquance sociale», par opposition à celui de «délinquance criminelle», est un synonyme de celui de conduite déviante.

Cet élargissement du contenu des conduites que la criminologie doit étudier va de pair avec les connaissances sur le syndrome général de la déviance. En effet, des théoriciens, comme Gottfredson et Hirschi (1990), considèrent toutes les formes de la conduite déviante comme assimilables au crime. Ce dont doutent d'autres théoriciens (Cullen, 1983) qui pensent qu'il faut considérer chaque forme de la conduite déviante indépendamment des autres formes. Par ailleurs, de nombreuses études empiriques, de Jessor et Jessor (1977), en passant par Osgood, Johnston et al. (1988), jusqu'à Le Blanc et Bouthillier (2003), démontrent clairement l'existence d'un syndrome comportemental qu'ils nomment «déviance générale». Ce syndrome comprend les diverses formes de l'activité délinquante, la consommation d'alcool et de drogues illicites, la promiscuité sexuelle, la rébellion familiale, l'inadaptation scolaire et la grossesse adolescente. Toutefois, l'existence de ce syndrome ne signifie pas que chacune des formes de la conduite déviante n'est pas analysable de façon indépendante (Le Blanc et Loeber, 1998).

Aujourd'hui, les théoriciens et les chercheurs, en criminologie et en psychologie, s'entendent pour reconnaître que les comportements déviants constituent un syndrome hiérarchique de conduites. Le Blanc et Bouthillier (2003) démontrent, à l'aide d'analyses factorielles confirmatoires, que la conduite déviante se décompose en conduites manifestes, clandestines, rebelles et imprudentes. Ces configurations s'expriment sous la forme de douze catégories de conduites déviantes. Chacune de celles-ci prend l'apparence de divers comportements. Cette conclusion 
est valide pour la participation, la fréquence, la variété, et l'âge d'initiation, tant pour les garçons que pour les filles. La figure 1 (page 406) illustre cette structure hiérarchique de la conduite déviante; cette figure doit être lue de droite à gauche. Le lecteur notera que les configurations clandestines et manifestes correspondent à la définition de la délinquance criminelle que propose le législateur canadien. Par ailleurs, les conduites rebelles et imprudentes se rapprochent soit de la délinquance statutaire, par exemple l'absentéisme scolaire, soit des troubles sérieux $\mathrm{du}$ comportement, par exemple la fugue ou la consommation abusive de substances psychoactives qui sont traitées en vertu de la Loi sur la protection de la jeunesse.

La conduite déviante est non seulement un syndrome, mais elle est aussi hétérotypique (Le Blanc et Loeber, 1998). Elle se manifeste avec continuité et changement à travers les phases de la vie. Au cours de l'enfance, elle apparaît surtout comme composée de conflits avec l'autorité et d'agressions physiques (Loeber et Hay, 1997). Au cours de l'adolescence, la gravité et la diversité des conduites déviantes précédentes augmentent et les conduites clandestines font leur apparition (vols divers), ainsi que les conduites imprudentes (Fréchette et Le Blanc, 1987; Le Blanc et Loeber, 1998). Tout au long de l'âge adulte, les conduites des phases antérieures disparaissent ou sont remplacées (le conflit avec l'autorité scolaire par le conflit avec les patrons) et de nouvelles conduites apparaissent (violence conjugale, évasion fiscale...).

La criminologie développementale décrit la conduite déviante sous la forme d'un $U$ inversé pour toutes les personnes au cours de leur vie. Ce dernier énoncé s'applique à toutes les formes et à tous les comportements qui composent la conduite déviante. Le Blanc et Loeber (1998) et Piquero et al. (2003) donnent des exemples de cette trajectoire normative pour diverses formes de la conduite déviante. Le cycle se manifeste, à une extrémité, par une conduite déviante d'une forme particulière d'un individu à un âge donné, par exemple le vandalisme à quinze ans. Ensuite, elle peut prendre la forme d'une séquence d'activités déviantes, plus ou moins nombreuses et variées, à différents âges. À l'autre extrémité, elle s'exprime par une continuité d'une grande variété d'activités déviantes sur plusieurs décennies, voire la plupart de la durée de la vie d'une personne. 
FIGURE 1

\section{La conduite déviante, structure hiérarchique}

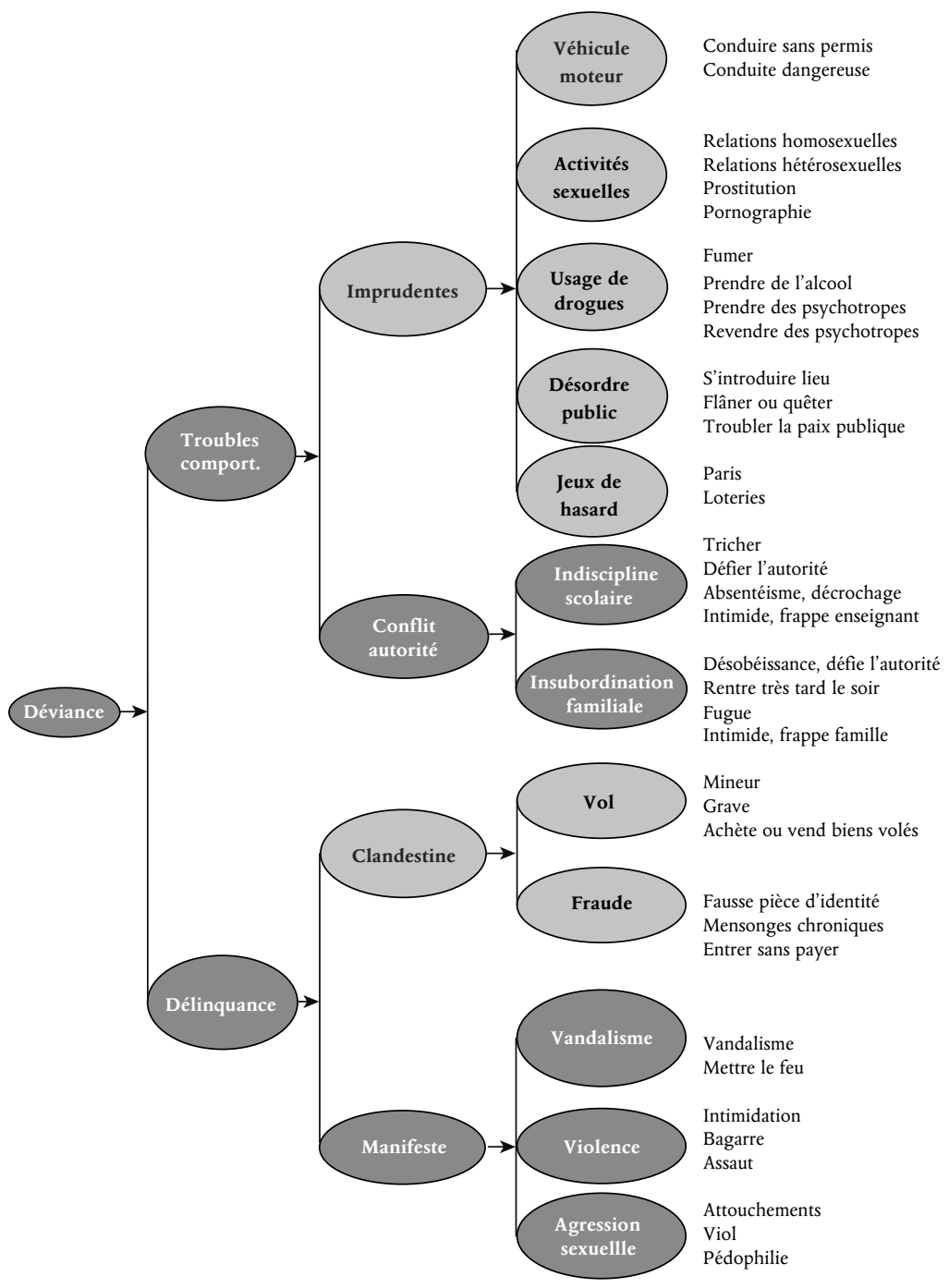

Le Blanc (2010). 


\section{Le cours de la conduite déviante}

Le paradigme développemental que proposent Le Blanc et Loeber (1998) distingue deux approches pour étudier l'enchaînement des activités déviantes dans la vie des personnes: l'étude des mécanismes des changements quantitatifs et l'étude des mécanismes des changements qualitatifs. Le tableau 1 présente les diverses manières de les apprécier.

TAB LEA U 1

Le cours de la conduite déviante: changements quantitatifs et qualitatifs

\begin{tabular}{|c|c|}
\hline Changements quantitatifs & Changements qualitatifs \\
\hline $\begin{array}{l}\text { Degré: La proportion des personnes } \\
\text { qui sont stables ou changent leur } \\
\text { participation, leur fréquence, } \\
\text { leur gravité. } \\
\text { Direction: La proportion des personnes } \\
\text { qui progressent ou qui régressent. } \\
\text { Vélocité: Le niveau de changement } \\
\text { à travers le temps sur la fréquence } \\
\text { (lambda), la gravité. } \\
\text { Développement: } \\
\text { - Activation: } \\
\text { - accélération: précocité et } \\
\quad \text { fréquence; } \\
\text { - diversification: précocité et } \\
\quad \text { variété; } \\
\text { - stabilisation: précocité et durée. } \\
\text { - Désistement: } \\
\text { - décélération: fréquence et arrêt; } \\
\text { - plafonnement: gravité et arrêt; } \\
\text { - spécialisation: variété et arrêt. }\end{array}$ & $\begin{array}{l}\text { Séquence développementale: } \\
\text { - Stades: une séquence des types } \\
\text { de comportements. } \\
\text { - Escalade: progresser sur les stades. } \\
\text { - Désescalade: régresser sur les stades. } \\
\text { - Cheminement: proportion des } \\
\text { personnes qui traversent un ou } \\
\text { plusieurs stades. } \\
\text { Conservation: } \\
\text { - Innovation: pratiquer un nouveau } \\
\text { comportement. } \\
\text { - Rétention: maintenir un comportement. } \\
\text { - Élimination: arrêter un comportement. } \\
\text { Synchronie entre les comportements: } \\
\text { - Simultanéité: deux comportements } \\
\text { évoluent en même temps. } \\
\text { - Décalage: deux comportements } \\
\text { n'évoluent pas en même temps. } \\
\text { - Adjacence: deux comportements sont } \\
\text { l'un avant ou après l'autre. } \\
\text { Trajectoires: Existe-il des tracés } \\
\text { d'évolution de chaque forme de la } \\
\text { conduite déviante? }\end{array}$ \\
\hline
\end{tabular}

Traduit de Le Blanc et Loeber (1998) et Le Blanc (2009).

\section{Les changements quantitatifs}

Les changements quantitatifs sont définis comme la mesure de la croissance et du déclin des activités déviantes. Premièrement, ils se manifestent par des modifications du niveau des activités: la participation et la fréquence de l'une ou l'autre des formes de l'activité déviante (Le Blanc 
et Loeber, 1998; Piquero et al., 2003, 2007). Deuxièmement, il peut s'agir de la direction du changement, progression ou régression de la fréquence, de la gravité ou de la variété (voir la revue des écrits de Le Blanc et Loeber, 1998). Troisièmement, la vélocité ou le degré de modification des paramètres de l'activité déviante est étudié (Le Blanc et Loeber, 1998; voir les écrits sur le lambda de Piquero et al., 2003). Quatrièmement, Le Blanc et Fréchette (1989) ont montré que le changement peut s'exprimer par l'accélération, la diversification et la stabilisation de l'activité délinquante. Finalement, selon ces auteurs, le déclin des activités déviantes s'opère par les mécanismes de la décélération, de la spécialisation et du plafonnement.

Le mécanisme d'activation décrit la manière dont le développement des activités déviantes est stimulé dès qu'il s'amorce et la manière dont sa persistance est assurée (Le Blanc et Fréchette, 1989; Le Blanc et Loeber, 1998). Les données permettent de soutenir que plus tôt un individu commence ses activités déviantes, plus abondantes, plus variées et plus durables elles seront. Les mécanismes d'accélération, de stabilisation et de diversification permettent de préciser comment les activités déviantes sont soumises à l'effet d'activation. La première poussée est celle de la stabilisation: la précocité s'affirme alors comme une source puissante pour assurer la durée. Les activités déviantes sont persistantes, mais elles ne sont pas nécessairement abondantes ou variées. La deuxième poussée est celle de l'accélération: l'apparition de l'agir déviant, soit au cours de la latence, soit au début de l'adolescence, entraîne une fréquence élevée. Les activités déviantes sont alors nombreuses, mais elles ne sont pas nécessairement variées ou durables. La troisième poussée est celle de la diversification: la précocité favorise un degré important de diversité. Les activités sont alors hétérogènes, mais elles ne sont pas nécessairement abondantes ou durables. Finalement, la quatrième poussée est la plus activante de toutes. Elle prend forme à partir de l'interaction entre la durée, la fréquence et la variété, trois mécanismes qui s'appuient sur la précocité. Les activités déviantes débutent tôt et deviennent, par la suite, abondantes, variées et durables, grâce à l'effet dynamique de leurs interactions. Les activités déviantes, quelles que soient leurs formes dans la structure hiérarchique du syndrome de la conduite déviante, se consolident et deviennent chroniques.

Le mécanisme de désistement décrit le cheminement de la conduite déviante qui précède son extinction. La conduite déviante, comme toutes les formes de sa structure hiérarchique, se construit, mais elle se 
déconstruit également. C'est le processus du désistement qui a été décrit pour la délinquance (Le Blanc et Fréchette, 1989; Le Blanc et Loeber, 1998). Le désistement est fonction de la durée, de la variété, de la gravité et de la fréquence de l'activité déviante. Ce qui veut dire que, plus la durée est prolongée, la fréquence élevée, la variété importante et la gravité forte, plus le désistement a des chances de se produire tard dans la vie des personnes. Trois mécanismes composent le processus de désistement: la décélération, la spécialisation et le plafonnement. La décélération met en cause le rapport entre la fréquence et le moment de l'arrêt de l'activité déviante. Au cours des années qui précèdent la cessation, la fréquence annuelle tend à diminuer progressivement. La spécialisation se rapporte à l'adoption progressive d'activités déviantes de moins en moins hétérogènes, de telle sorte que, plus approche l'âge d'arrêt, plus le degré de variété diminue. Le plafonnement fait référence à la situation du déviant qui a atteint le niveau le plus élevé de gravité d'agir déviant pour lui; l'atteinte de ce sommet est le présage d'une cessation imminente.

Le Blanc (2005a, 2009) soutient que les mécanismes quantitatifs de la croissance et du déclin s'appliquent à toutes les formes du syndrome de la conduite déviante.

\section{Les changements qualitatifs}

Les changements qualitatifs sont définis de deux manières. D'une part, la criminologie s'est toujours demandé s'il existe un mécanisme d'aggravation et d'atténuation des activités déviantes. D'autre part, Le Blanc et Loeber (1998) ont introduit d'autres méthodes pour définir les changements qualitatifs.

L'aggravation fait référence à l'existence d'une séquence d'apparition des diverses formes de la conduite déviante qui s'échelonne des conduites bénignes aux activités les plus graves à mesure que l'âge s'accroît (Le Blanc et Loeber, 1998). La reconnaissance d'une telle séquence représente une sorte de patron standard de développement par lequel passent les personnes qui s'orientent vers une conduite déviante répétitive. L'étude du mécanisme d'aggravation se fait par le truchement de deux grilles de lecture. La première grille, qui focalise sur les conduites déviantes elles-mêmes, s'efforce de dégager un enchaînement spécifique parmi ces dernières. La seconde grille, qui s'intéresse aux personnes, vise à faire apparaître un mode progressif d'implication dans la conduite 
déviante qui met en cause le passage à travers les stades d'évolution de la conduite déviante. Une bonne partie de la confusion dans laquelle s'empêtrent les écrits criminologiques résulte du manque de différenciation entre ces deux perspectives complémentaires (Le Blanc et Fréchette, 1989).

Le Blanc et Fréchette (1989) ont décrit des stades de développement de l'activité délinquante à l'aide des formes de cette dernière, de leur précocité, de leur gravité, de leur durée et de l'âge: apparition, exploration, explosion, conflagration et débordement. Au départ, habituellement entre huit et dix ans, les activités délictueuses sont homogènes et bénignes. Elles s'expriment à peu près strictement sous la forme de menus larcins; il s'agit du stade de l'apparition. Par la suite, les essais se continuent, généralement entre dix et douze ans, par une diversification et une aggravation des délits qui se manifestent par le vol à l'étalage et le vandalisme; il s'agit du stade de l'exploration. Ultérieurement, autour de treize ans, une augmentation substantielle de la variété et de la gravité des délits apparaît et quatre nouveaux types de délits prennent leur essor; ce sont le vol simple, les désordres publics, le vol avec effraction et le vol sur une personne. Il s'agit du stade de l'explosion avec, comme épine dorsale, le vol avec effraction qui, à cause de sa longévité plus importante, constitue le ferment majeur de cette nouvelle expansion. Ensuite, autour de quinze ans, l'hétérogénéité, la variété et la gravité augmentent encore. Quatre types de délits viennent étoffer cette amplification, soit le commerce des substances psychoactives, le vol d'un véhicule à moteur, le vol grave et l'attaque d'une personne; il s'agit du stade de la conflagration. Le cinquième stade, qui se manifeste uniquement au cours de l'âge adulte, est celui du débordement vers des formes plus astucieuses ou plus violentes d'agir délictueux.

Des stades d'aggravation sont connus pour la consommation de drogues (Kandel, 2002; Le Blanc, 2005b), la violence (Loeber et Hay, 1997; Le Blanc, 1999) et l'enchaînement d'une grande variété d'activités déviantes (Le Blanc et Girard, 1998). Dans le domaine de la consommation de psychotropes, Kandel (2002) propose quatre stades hiérarchiques du développement de cette conduite. Ce sont, dans l'ordre, la consommation de bière ou de vin, la consommation de cigarettes, la consommation de marihuana et l'expérimentation d'autres drogues illicites. Depuis ces travaux, plusieurs recherches ont confirmé l'existence de stades dans le développement de la consommation de psycho- 
tropes et elles ont spécifié la séquence développementale en intégrant d'autres psychotropes comme la cocaïne, les stimulants, les hallucinogènes et la revente de psychotropes (Le Blanc et Girard, 1998). En conséquence, l'expérimentation de l'alcool est suivie par celle des drogues «douces» (marihuana et dérivés du haschich), des drogues de synthèse (hallucinogènes et stimulants) et des drogues «dures» (cocaïne et héroïne).

Le domaine de la violence interpersonnelle ne fait pas exception (Le Blanc, 1999). La séquence d'aggravation des comportements violents va des agressions impulsives (se battre, se fâcher, être bousculé et frapper) à des comportements qui ajoutent un danger potentiellement grave pour la victime parce qu'ils impliquent l'utilisation d'une arme (lancer des objets, porter une arme, utiliser une arme pour se battre). Viennent ensuite des comportements qui sont plus sophistiqués parce qu'ils comprennent l'intimidation (encourager à s'en prendre à quelqu'un, menacer pour faire exécuter quelque chose, menacer ou malmener pour l'obtenir). La séquence d'aggravation se termine par des comportements graves par leur gratuité (battre quelqu'un qui ne m'a rien fait), par la proximité de la victime (agresser physiquement un parent) et par le traumatisme et les conséquences psychologiques et physiques impliquées (agression sexuelle). Loeber et al. (1997) confirment l'existence des deux premiers stades: agressions impulsives et comportement potentiellement dangereux.

Pour leur part, Le Blanc et Girard (1998) ont vérifié l'enchâssement d'un large éventail de conduites déviantes. D'abord, quatre formes apparaissent dans le répertoire comportemental avant onze ans. Il s'agit des conflits avec l'autorité scolaire et les parents, des vols mineurs et des agressions sur une période d'une année et demie. Il faut ensuite attendre environ une année avant de voir s'enrichir le répertoire des conduites déviantes. Le répertoire s'élargit au cours de la douzième année avec deux nouvelles conduites, la consommation des psychotropes et les relations sexuelles, la première précédant de peu les secondes. Finalement, les adolescents passent aux manifestations les plus graves de la conduite déviante, c'est-à-dire la prostitution, l'agression sexuelle et les vols graves, entre treize et quinze ans. Cette séquence d'initiation des types de conduites déviantes correspond bien au modèle que Loeber et al. (1997) proposent. La conduite déviante démarre par des conflits avec l'autorité familiale et scolaire. Elle enchaîne avec la délinquance mineure, le vol et le vandalisme, ou les agressions. Finalement, ces 
auteurs suggèrent que la conduite déviante se diversifie vers la délinquance acquisitive grave ou de violence. Par contre, ces auteurs ne tiennent pas compte de la consommation de substances psychoactives qui prend fermement position avant l'apparition de la délinquance grave.

Jusqu'ici, l'aggravation a été analysée comme une séquence de comportements de plus en plus graves. Une deuxième manière de l'aborder consiste à mesurer si les personnes progressent à travers les stades de l'aggravation. Par exemple Le Blanc et Fréchette (1989) observent que $92 \%$ des délinquants changent de stades lorsque les cinq stades de développement de l'activité délictueuse sont envisagés. Selon ces auteurs, $84 \%$ des délinquants progressent vers une délinquance plus grave, alors que seulement $16 \%$ des instables régressent vers des formes moins nocives d'activités criminelles. Il convient donc de conclure que toutes les séquences développementales des conduites déviantes sont définies comme hiérarchiques plutôt qu'embryogéniques. Elles sont embryogéniques si toutes les personnes commencent au même stade et si elles traversent tous les stades. Elles sont plutôt hiérarchiques parce que les personnes commencent à divers stades et elles ne progressent qu'à travers certains stades. L'aggravation se façonne donc par l'innovation, l'introduction d'un nouveau comportement déviant, la rétention, le maintien d'un comportement, et l'élimination, la disparition d'un comportement existant. L'aggravation est aussi synchronique par la simultanéité temporelle ou par un décalage des formes d'activités déviantes et d'enchâssement temporel de différentes formes d'activités déviantes (voir par exemple: Le Blanc et Kaspy, 1998, et Le Blanc et Girard, 1998).

En somme, l'hypothèse de l'aggravation des diverses formes de l'activité déviante est un fait de plus en plus solide. Toutefois, les mécanismes de l'atténuation de l'activité déviante sont beaucoup moins bien documentés même si les données sont encourageantes (Le Blanc, 2002). Par exemple, l'atténuation de la consommation de substances psychoactives suit la route inverse de l'aggravation: les personnes passent de la consommation de drogues «dures» à celle de drogues de synthèse, puis aux drogues «douces» et à l'alcool (Le Blanc, 2005b). Le cheminement de l'atténuation des activités délinquantes est inversé par rapport à celui de l'aggravation pour les activités délinquantes, c'est-à-dire le passage des activités les plus graves aux moins graves, par exemple des activités violentes aux activités non violentes (Le Blanc et Fréchette, 1989). 


\section{Les trajectoires de la conduite déviante}

Les processus d'activation, d'aggravation et de désistement décrivent la construction et la déconstruction de la conduite déviante durant la vie des personnes. Ce cycle prend la forme de trois méta trajectoires. Les chercheurs les reconnaissent pour diverses formes de la conduite déviante selon les recensions de Le Blanc et Loeber (1998), Le Blanc (2002) et nos travaux récents (Le Blanc, 2010). Ces trajectoires se manifestent de la manière suivante pour la conduite délinquante (Fréchette et Le Blanc, 1987; Le Blanc et Fréchette, 1989; Le Blanc, 1995 ; Lanctôt et Le Blanc, 2000). Il s'agit des conduites délinquantes d'occasion, de transition et de condition. En criminologie, il est courant de faire référence, pour les deux dernières méta trajectoires, à la délinquance limitée à l'adolescence («adolescence-limited») et à la délinquance qui persiste au cours de la vie («life-course persistent») selon les dénominations proposées par Moffitt (1993) (voir une synthèse critique récente des écrits: Skardhamar, 2009).

La conduite délinquante d'occasion ou la délinquance commune est le fait de $45 \%$ des adolescents. Il s'agit d'une conduite délinquante tout à fait insignifiante, c'est-à-dire quelques infractions d'une nature mineure (vol à l'étalage, vandalisme, vols mineurs, désordres publics); ces quelques infractions sont soit concentrées sur une période de temps limitée, soit échelonnées sur la totalité de l'adolescence. Elle est le fait des filles autant que des garçons et elle se manifeste dans toutes les classes sociales (Fréchette et Le Blanc, 1987; Le Blanc, 1995). Il s'agit de $9 \%$ des actes délinquants et de $16 \%$ des délinquants qui sont arrêtés par les services de police (Le Blanc, 1995).

La conduite délinquante de transition se distingue de la conduite occasionnelle en ce qu'elle affirme un degré supérieur de gravité, de durée, de volume, de diversité. Elle s'observe chez $45 \%$ des adolescents et elle se présente sous la forme d'une durée plus longue, à savoir quelques années. Elle est d'un volume plus élevé (annuellement trois à cinq délits) et d'une gravité plus forte (quelques fois le vol avec effraction). Chez les pupilles du tribunal, elle s'exprime sous une forme encore plus aggravée. Il s'agit d'un nombre limité de conduites, d'une gravité variable, mais qui se limite à la période du début ou du milieu de l'adolescence (Fréchette et Le Blanc, 1987). En fait, la conduite délinquante de transition apparaît essentiellement comme une crise du milieu de l'adolescence. Cette forme d'activités délinquantes correspond 
à environ $40 \%$ des actes rapportés par les adolescents et à environ $25 \%$ des actes connus des services de police (Le Blanc, 1995).

La dernière trajectoire, la conduite de condition, se caractérise avant tout par la persistance et l'aggravation des délits. Elle débute tôt, autour de dix ans. La personne progresse des infractions mineures (les vols à l'étalage et les larcins) vers les délits majeurs (les vols avec effraction ou les délits graves contre la personne), cela avant l'âge de quinze ans. Les actes sont nombreux et hétérogènes. Cette conduite délinquante persistante se présente, à la fin de l'adolescence, sous un mode mineur qui ne s'aggrave pas au point de présenter des délits contre la personne, mais qui comprend de multiples délits contre les biens, particulièrement des vols avec effraction. Sous son mode majeur, c'est la conduite qui, tout en étant volumineuse, hétérogène, continue, précoce, est déjà, à quinze ans, aussi grave que la conduite délinquante adulte sérieuse: vols sur la personne, vols à main armée, etc. Cette forme de la conduite délinquante se retrouve chez $5 \%$ de la population. Elle correspond à plus de $50 \%$ des actes rapportés par les adolescents et à plus de $60 \%$ des actes connus des services de police (Le Blanc et Fréchette, 1989; Le Blanc, 1995). Les délinquants persistants judiciarisés sont responsables d'au moins $50 \%$ des actes délinquants et des deux tiers des délits avec violence (Le Blanc, 1995).

Ces méta trajectoires rendent bien compte de toutes les trajectoires reconnues par différentes méthodes (Le Blanc, 2002). Depuis la fin des années 1990, des méthodes statistiques complexes sont utilisées pour rechercher des trajectoires développementales de la conduite délinquante (Piquero et al., 2003, 2007; Skardhamar, 2009), d'autres formes de la conduite déviante (Le Blanc et al.,, 2002), et des liens ont été faits avec les trajectoires des troubles du comportement (Le Blanc et Kaspy, 1998; Le Blanc, 2010, inédit). L'équipe du Groupe de recherche sur l'inadaptation psychosociale a été particulièrement active dans ce type de recherche: agression physique et violence (Nagin et Tremblay, 1999; Loeber et al., 2006), participation à un gang (Lacourse et al., 2003), délinquance et comportement turbulent (Broidy et al., sous presse), jeu de hasard (Vitaro et al., 2004), drogue (Wanner et al., 2006). Dans ces travaux, plus de quatre trajectoires sont dégagées et elles se rassemblent sous les trois méta trajectoires proposées. En somme, toutes les formes de la conduite déviante qui composent son syndrome se manifestent par ces méta trajectoires dans la vie des personnes. Elles sont aussi composées de trajectoires secondaires (Le Blanc et Fréchette, 1989; Le Blanc, inédit). 


\section{L'autorégulation de la conduite déviante}

Aujourd'hui, la criminologie est équipée pour décrire le cycle de toutes les formes de la conduite déviante. Les paramètres descriptifs font consensus parmi les chercheurs. Les mécanismes de son apparition, de son développement et de son déclin sont spécifiés pour les activités délinquantes et, de mieux en mieux, pour les autres formes de la conduite déviante. Par ailleurs, la psychologie du développement et le paradigme de l'ordre et du chaos en science amènent la criminologie à se demander dans quelle mesure la mécanique de l'autorégulation des phénomènes s'applique au cycle de la conduite déviante (pour plus de détails: Le Blanc, 2005a, 2009).

Le paradigme de l'ordre et du chaos (Gleick, 1987; Briggs et Peat, 1989) s'appuie sur plusieurs principes qui sont transcrits de la manière suivante pour la conduite déviante. Le système que constitue le syndrome de la conduite déviante est ouvert à diverses influences (les facteurs biologiques, psychologiques et sociaux qui caractérisent la personne: voir Le Blanc, 1997, 2006). Ce système est complexe et fractal parce qu'il se compose d'une hiérarchie de manifestations qui sont de plus en plus fines, de la conduite déviante dans son ensemble à des comportements spécifiques comme le vol d'une automobile, la consommation d'une «ligne» de cocaïne, etc. Ce système s'auto-organise pour conserver son énergie selon son rythme interne, c'est-à-dire que les mécanismes de l'apparition, du développement, du maintien et du déclin des formes de la conduite déviante ne se modifient pas, ou les changements se compensent de l'un à l'autre. L'autorégulation de la conduite déviante s'accomplit selon une dynamique qui n'est pas linéaire et la coévolution des cycles des différentes formes de la conduite déviante est évidente. Le développement du système que constitue le syndrome de la conduite déviante obéit aussi à plusieurs principes que le paradigme de l'ordre et du chaos et la psychologie du développement partagent. Il y a d'abord le principe de l'orthogenèse qui dit que la direction du développement est celle de l'augmentation de la complexité d'un phénomène, ainsi la conduite déviante se manifeste par deux formes au cours de l'enfance (désobéissances et agressions) et par de plus en plus de formes au cours de l'adolescence (les conduites imprudentes, de conflit avec l'autorité, clandestines et manifestes). Il y a ensuite le principe de la sensibilité aux conditions initiales de la conduite déviante (l'effet de la précocité sur la continuité de la conduite 
est bien connu pour l'activité délinquante selon les recensions des écrits de Le Blanc et Loeber, 1998, et Piquero et al., 2003). En outre, il y a le principe de la probabilité épigénétique ou du hasard structuré selon lequel le cycle de la conduite déviante obéit à un hasard organisé par le processus du développement de la conduite. Le dernier principe à mentionner est l'interdépendance réciproque entre les formes de la conduite déviante, c'est-à-dire que les diverses formes de cette dernière s'alimentent les unes les autres.

Jusqu'ici, il a été proposé que le cours de la conduite déviante des personnes s'appréhende par la description des changements quantitatifs et qualitatifs qui la façonnent. Ces instruments ne permettent pas de répondre à la question suivante: comment sont produits ces changements quantitatifs et qualitatifs pendant le cycle de la conduite déviante? Cette question introduit la notion de l'existence d'un processus développemental qui se manifeste par les changements quantitatifs et qualitatifs dans l'activité déviante. Ces changements ne sont pas le processus lui-même. Lapplication des principes ci-dessus et des instruments géométriques du paradigme de l'ordre et du chaos (Briggs et Peat, 1989) permettent de formuler des hypothèses sur la mécanique du cycle de la conduite déviante (Le Blanc, 2005a, 2006, 2009).

\section{Attracteurs et répulseurs}

Un attracteur est un élément magnétique qui structure un phénomène. La figure 2 (page 418), décrit deux attracteurs, l'activité délinquante et la consommation de substances psychoactives. Le caractère magnétique d'un attracteur provient de la tendance d'un comportement à se répéter une fois qu'il a été émis pour une première fois. Il est bien connu que la très grande majorité des adolescents, plus de $80 \%$ selon Fréchette et Le Blanc (1987), ont l'occasion de pratiquer des comportements délinquants et de consommation de substances psychoactives, surtout les plus bénins comme le vandalisme et la consommation de marihuana. Chacun de ces attracteurs n'a pas la même puissance selon l'âge, le sexe, le milieu de l'adolescent et ses caractéristiques biologiques, psychologiques et sociales. La force d'un attracteur dépend, au point de départ, de ses paramètres et de son degré de liberté dans le système de la conduite déviante. Par exemple, les paramètres de la consommation d'alcool sont la nature du produit (bière ou alcool), la quantité (un verre 
ou plusieurs), la durée (quinze minutes ou deux heures) et la fréquence (une fois par semaine ou tous les jours). Le degré de liberté résulte de la combinaison de ces paramètres pour une personne donnée. En conséquence, si la personne prend plusieurs verres d'alcool pendant quinze minutes et plusieurs fois par semaine, sa probabilité de répéter ce comportement est maximale. À l'inverse, si la personne prend un verre de bière sur une période de deux heures et une fois par semaine, sa probabilité de répéter ce comportement est minimale.

Certains comportements sont des répulseurs pour une personne donnée. Ces comportements inspirent de la répulsion à une personne et celle-ci est donc portée à s'en abstenir. Par exemple, faire un vol avec effraction ou consommer des drogues de synthèse peuvent être des répulseurs pour une personne et des attracteurs pour une autre personne. Pour une personne, faire un vol avec effraction inspire de la peur et l'ingurgitation d'une drogue chimique de la répulsion, du dégoût. Pour certaines personnes, tous les comportements qui composent le syndrome de la conduite déviante sont des attracteurs, tandis que pour d'autres, ce sont des répulseurs. Ils constituent une source d'équilibre ou de chaos pour une personne compte tenu de l'état de ses liens sociaux et de sa maîtrise de soi (Le Blanc, 2006).

La figure 2 illustre la présence de plusieurs types d'attracteurs ou répulseurs. Abraham (1995) les définit de la manière suivante. Il s'agit d'abord des attracteurs nodaux (section a de la figure 2: la précocité d'une conduite déviante, par exemple la première consommation d'un «joint» de marihuana). Si le comportement se répète, l'attracteur gagne en puissance et il est qualifié d'attracteur périodique (section b de la figure 2); la consommation de marihuana augmente. L'attracteur périodique devient une spirale de comportements (section c de la figure 2), si, par exemple, la consommation de marihuana passe d'une fois par semaine à plusieurs fois par semaine. Ensuite, l'attracteur peut devenir cyclique (section d de la figure 2), par exemple si la consommation devient journalière. L'exemple du «joint» de marihuana s'applique à toutes les formes de la structure hiérarchique de la conduite déviante.

La figure 2 illustre aussi qu'avec le temps, d'autres attracteurs s'ajoutent (par exemple le vol, comme attracteur nodal, à la section e de la figure 2). Non seulement le développement de la conduite déviante gagne ainsi en diversification, mais un nouvel attracteur peut gagner en puissance en devenant périodique, spirale et cyclique (les sections $\mathrm{f}$ et $\mathrm{g}$ de la figure 2). Avec le temps, le système peut voir son niveau 
FIGURE 2

Un exemple de l'autorégulation de la conduite déviante: marihuana et vol; adapté de Abraham (1992) et Le Blanc (2009)

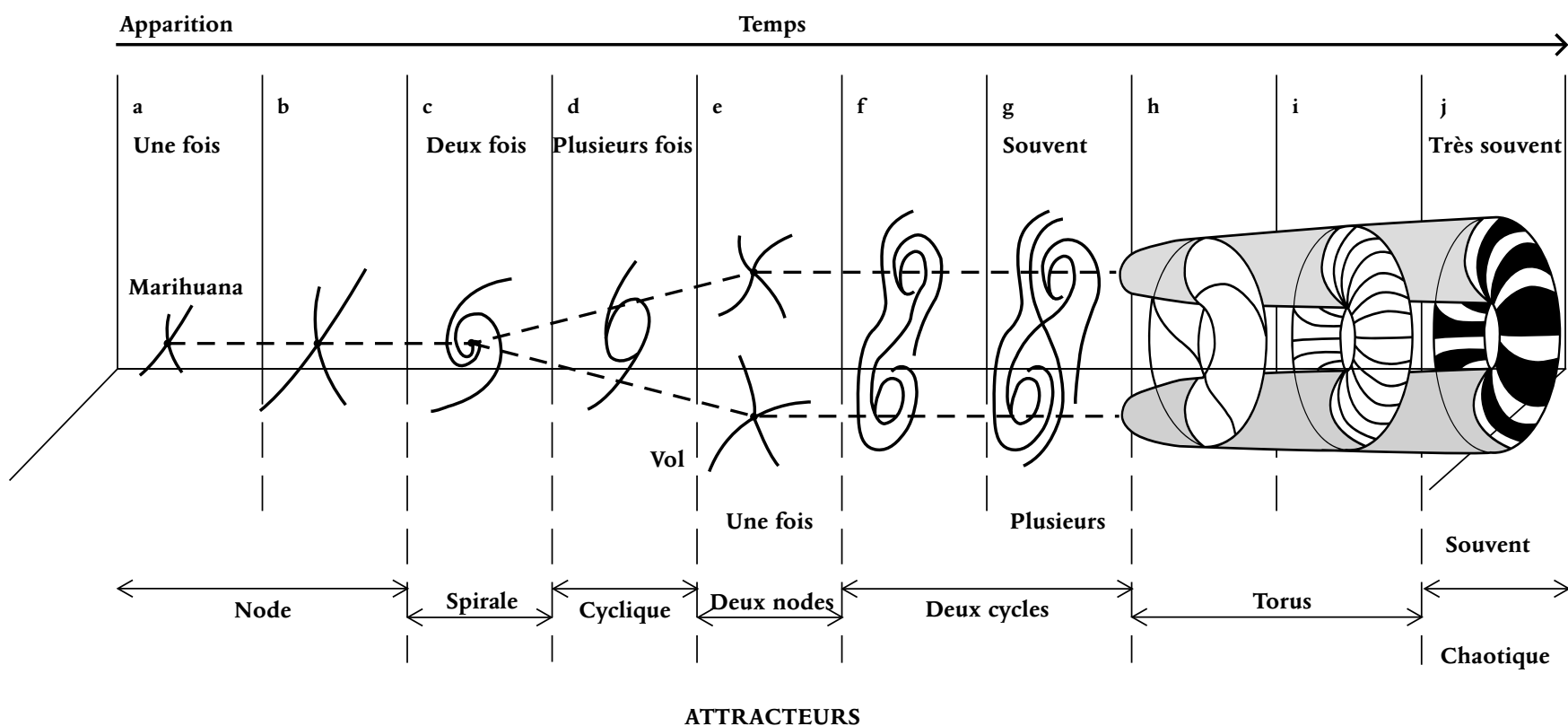


d'incertitude augmenter et il devient alors difficile, sinon impossible, de prédire quelle conduite déviante sera produite. Cet état est appelé l'attracteur étrange (la section à l'extrême droite de la figure 2). Il s'agit de l'état d'une personne qui consomme de la marihuana en grande quantité chaque jour et qui commet une grande variété de vols régulièrement, alors il y a chaos ou la certitude qu'un autre comportement sera produit, mais une incertitude quant à savoir quel sera le prochain comportement déviant produit. De plus, il est probable qu'un nouvel attracteur nodal apparaisse, le conflit avec l'autorité scolaire par exemple. Ce cheminement pourrait constituer une méta trajectoire de la conduite déviante de transition. Si les comportements déviants augmentent encore en diversité, gravité, durée, etc., alors la personne se situe sur la méta trajectoire de la conduite déviante persistante; elle serait une toxicomane, un délinquant persistant.

Il va de soi que le cheminement d'aggravation de la conduite déviante, qui va d'un attracteur nodal vers un attracteur étrange, a la possibilité de s'interrompre sous l'effet de la saturation comportementale, de la consolidation des liens sociaux et de la maîtrise de soi ou des deux mécanismes. Les attracteurs deviennent alors des répulseurs et la personne, subitement ou progressivement, fait le chemin inverse de celui décrit à la figure 2; c'est le désistement (Le Blanc, 2005a).

\section{Les bifurcations}

Les changements qualitatifs dans le développement de la conduite déviante se produisent sous la forme d'une aggravation. Ce mécanisme se présente sous la forme d'une progression à travers des stades. Leur existence suppose une période critique à l'intersection de deux stades. Cette période s'appelle transition en psychologie développementale ou apprentissage pour les comportementalistes (Lerner, 2002). Pour les criminologues, il s'agit d'embranchements (Sampson et Laub, 1993) ou de dérives (Matza, 1957). Pour les théoriciens du chaos, il s'agit de bifurcations (Gleick, 1987).

Une bifurcation est le choix d'un chemin différent en raison de modifications des paramètres et du degré de liberté qui régularisent un attracteur ou un répulseur. Il s'agit d'un changement majeur dans l'état du système de la conduite déviante. À la section e de la figure 2, un changement a lieu parce que le système de la conduite déviante était uniquement composé de la consommation de marihuana, alors qu'il 
comprend maintenant une autre forme de conduite déviante, le vol. Le bassin d'attracteurs est ainsi augmenté, tout comme le nombre de paramètres et de degrés de liberté. L'incertitude augmente alors dans le système, le chaos progresse. Abraham (1995) propose plusieurs types de bifurcations que Le Blanc (2006) applique à la conduite déviante. Avant et après une bifurcation, le système de la conduite déviante est stable, la probabilité d'apparition d'une conduite est constante; il y a de l'ordre dans le système. Par contre, au moment de la bifurcation, la probabilité d'une conduite déviante, consommation de marihuana ou vol, est incertaine, le chaos caractérise le système de la conduite déviante.

\section{L'autorégulation de la conduite déviante}

Lautorégulation est une caractéristique fondamentale de tous les êtres vivants et de tous les humains. Il s'agit d'un principe accepté par les spécialistes de la psychologie du développement (Lerner, 2002) et du paradigme de l'ordre et du chaos (Gleick, 1987). Pour les psychologues, tous les humains sont actifs dans leur développement. Ils activent et désactivent leur environnement. Pour les spécialistes de l'ordre et du chaos, les humains peuvent contrôler les paramètres de leurs comportements et faire bifurquer, même inverser, la direction de leur système comportemental, donc modifier la nature de leur conduite déviante. Les personnes ne sont pas obligées d'expérimenter la consommation de marihuana ou le vol, ou de les aggraver.

Quelle que soit l'importance de l'autorégulation en comparaison de l'impact des liens sociaux et de la maîtrise de soi, un système de conduite déviante tend à devenir de plus en complexe (les sections a à j de la figure 2). Compte tenu de cette complexité, il existe diverses formes d'équilibre dans le système (Briggs et Peat, 1989). Une première forme est celle de l'équilibre chaotique par lequel l'évolution du système est irréversible. L'exemple typique de cet état est celui de la toxicomanie dont le traitement a peu de chances de renverser cet état de la personne. Une autre forme d'équilibre est celle de l'émergence spontanée de l'ordre, la conduite déviante est alors réversible; le vol et la consommation de marihuana sont abandonnés. Les trajectoires du désistement de la criminalité de Laub et al. (1998) illustrent ces formes de changements. L'équilibre chaotique est représenté par les délinquants persistants à faible, moyenne et haute fréquence, tandis que les désisteurs à fréquence 
modérée et les classiques (l'activité criminelle s'arrête au cours de l'adolescence) illustrent l'émergence spontanée de l'ordre.

Il est possible d'illustrer l'autorégulation à partir d'une étude empirique qui porte sur la précocité et la variété de l'activité délinquante rapportée et de la consommation des substances psychoactives sur une période de 25 ans, grâce aux données de l'Étude longitudinale montréalaise avec deux échantillons (Le Blanc, 2009). La figure 3 (page 422), illustre que l'autorégulation s'accomplit à travers trois types de liens entre les variables qui représentent la conduite déviante. Il s'agit des liens du lancement, ceux qui proviennent du moment de l'apparition de la conduite déviante (un attracteur nodal), des liens contemporains, les interactions entre des formes de la conduite déviante à un âge donné (un attracteur cyclique), et des liens causaux, l'effet de la conduite précédente sur la conduite subséquente (la probabilité de répéter un comportement: ses paramètres et son degré de liberté).

Le test empirique d'un modèle de l'autorégulation est limité à deux formes de la conduite déviante, l'activité délinquante et la consommation des substances psychoactives. Il est réalisé avec un échantillon d'adolescents judiciarisés qui a été suivi longitudinalement pendant 25 ans et avec l'aide de la technique de l'analyse des cheminements de la causalité. Les observations statistiquement significatives suivantes en sont extraites (voir les flèches à la figure 3) (Le Blanc, 2009). La conduite déviante à un âge prédit la conduite à un âge subséquent. Cette continuité à travers les âges est plus importante pour la consommation des drogues que pour l'activité délinquante. Un effet de lancement existe puisque la précocité prédit le niveau de l'activité délinquante et de la consommation de drogues à 15 ans et pas par la suite. À chaque âge, l'activité délinquante est associée à la consommation de drogues et l'inverse. Ces deux formes de conduite déviante se soutiennent l'une et l'autre. Par ailleurs, la diminution de la consommation des drogues à partir de 30 ans encourage la diminution des activités délinquantes à partir de ce moment-là (Morizot et Le Blanc, 2007). Les effets croisés dans le temps sont extrêmement rares, par exemple de la consommation de drogues à 15 ans sur l'activité délinquante à 30 ans et l'inverse. En somme, la conduite déviante se construit d'abord d'un effet de lancement qui se transforme ensuite en continuité et celle-ci est soutenue par les interactions contemporaines entre les conduites déviantes.

Cette exploration du phénomène de l'autorégulation avec seulement deux formes de conduite déviante confirme la mécanique décrite et 
FIGURE 3

L'autorégulation de la conduite déviante: modèle théorique et exemple empirique (flèches en gras) avec l'activité criminelle et la consommation de drogues

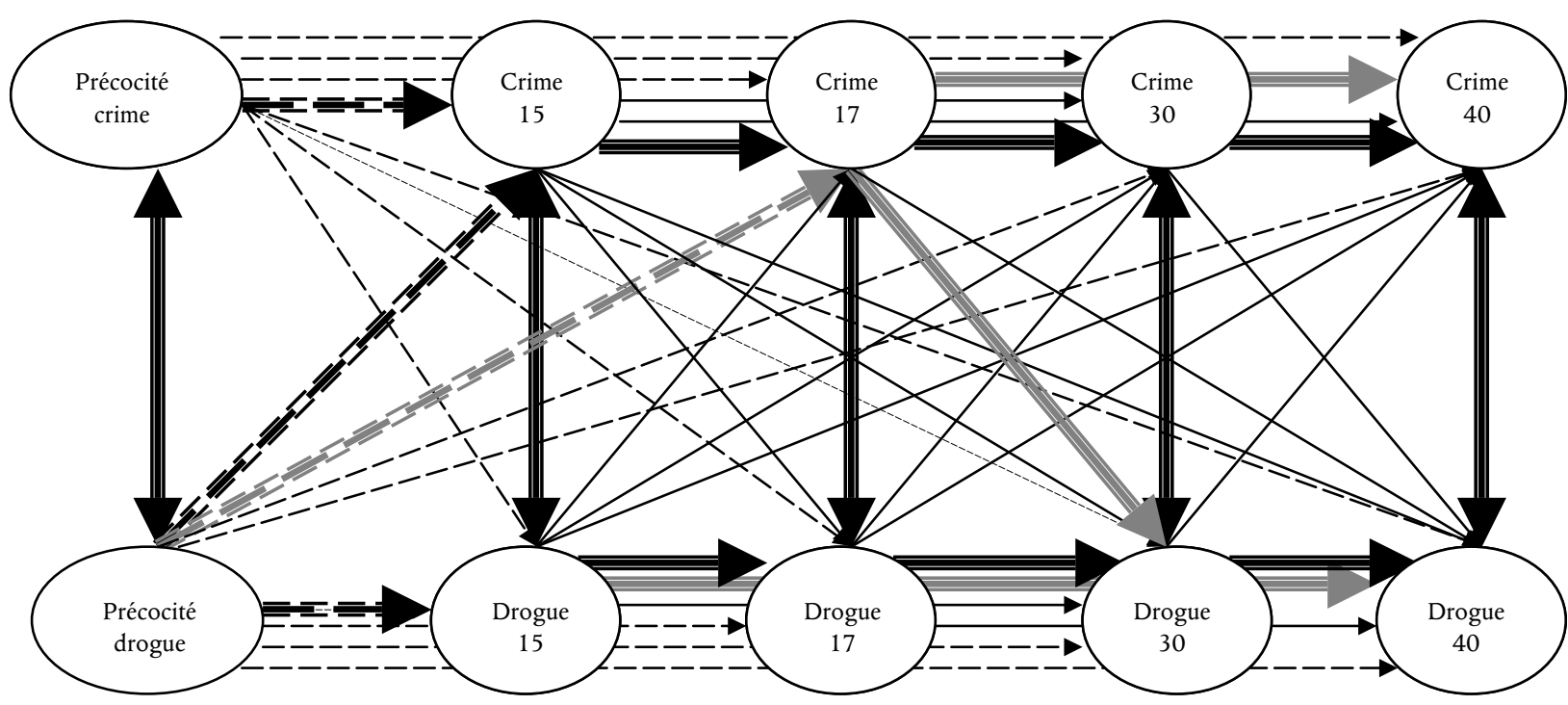

Légende

Effet de lancement $-\longrightarrow-\longrightarrow$

Effet contemporain

Effet causal

Chemins statistiquement significatifs

Traduit de Le Blanc (2009). 
montre qu'il s'agit d'un phénomène extrêmement complexe compte tenu de la composition du syndrome hiérarchique et cyclique de la conduite déviante. Plusieurs travaux confirment indirectement la mécanique de l'autorégulation par l'étude de l'entrecroisement des trajectoires de diverses formes de la conduite déviante: accidents, vols, agressions, jeu, vandalisme, drogues (Dembo et al., 2007; Begg et Guilliver, 2008; McDonald et al., 2009; Wanner et al., 2009; van Lier et al., 2009). Le paradigme de l'ordre et du chaos a permis une conceptualisation plus précise du processus de construction de la conduite déviante d'une personne. Il permet à la criminologie de dépasser la description quantitative et qualitative du cours de la conduite déviante et de mieux comprendre le processus développemental sous-jacent.

\section{Conclusion}

Le paradigme développemental a pris forme, d'abord, par l'introduction des notions d'activation, d'aggravation et de trajectoires dans nos travaux à partir de 1980 (Fréchette et Le Blanc, 1987). Ensuite, il s'est élaboré par la démonstration de l'existence des mécanismes d'activation, d'aggravation et de désistement (Le Blanc et Fréchette, 1989). Plus tard, la formalisation du paradigme développemental par des revues, des écrits lui a donné ses lettres de noblesse (Loeber et Le Blanc, 1990; Le Blanc et Loeber, 1998). Finalement, la conception et la validation de la mécanique de l'autorégulation ont complété le contenu de ce paradigme (Le Blanc, 2005a, 2009).

La question de la mécanique des processus développementaux pose un défi majeur à la criminologie par la complexité qu'elle représente. Il lui est facile de chiffrer les limites de la carrière criminelle pour divers paramètres (âge du début et de la fin, durée, fréquence). Il lui est possible d'exposer, avec des outils statistiques, les mécanismes de l'activation, de l'aggravation et du désistement. Par contre, il lui est difficile de représenter la mécanique du développement du syndrome de la conduite déviante qui comprend une douzaine de types de comportements. Les outils du paradigme de l'ordre et du chaos ont permis de progresser dans la conception de cette mécanique. Toutefois, il existe encore un décalage énorme entre la formulation discursive de la mécanique de l'autorégulation, son opérationnalisation et sa vérification empirique. Néanmoins, les données actuelles permettent d'entrevoir un peu de lumière. Par contre, il faut reconnaître que l'utilité heuristique 
de l'intégration des notions de la psychologie du développement et du paradigme de l'ordre et du chaos permet d'approfondir la compréhension du développement du syndrome de la conduite déviante.

Si l'adoption du paradigme développemental apparaît fructueuse pour la criminologie de la conduite déviante, une question demeure. Le paradigme développemental s'applique-t-il à la compréhension des causes de la conduite déviante? Le Blanc (2006) montre, comme pour la conduite déviante, que les liens sociaux et la maitrise de soi se déploient dans une structure hiérarchique, que le développement de ces causes peut se décrire en termes de changements quantitatifs et qualitatifs. Dans le domaine des liens sociaux, les connaissances développementales sont rudimentaires. Dans le domaine de la maîtrise de soi, il existe des mécanismes de bonification et d'immobilisme et des trajectoires (Morizot et Le Blanc, 2003, 2005). Le Blanc (2006) montre que la mécanique de l'autorégulation pourrait s'appliquer au développement interactif de la conduite déviante, des liens sociaux et de la maîtrise de soi. Dans ce dessein, il utilise la figure 2 où il remplace la consommation de marihuana et le vol par la conduite déviante et il rajoute les liens sociaux et la maîtrise de soi.

\section{Références}

Abraham, F. D. (1992). Visual introduction to dynamical systems theory for psycho$\log y$. Santa Cruz: Aerial Press.

Abraham, F. D. (1995). Introduction to dynamics: A basic language: a basic metamodeling strategy. In F. D. Abraham \& A. R. Gilgen (Eds.), Chaos theory in psychology (28-42). Wesport: Greenwood Press.

Begg, D. J., \& Guilliver, P. (2008). A longitudinal examination of the relationship between adolescent problem behavior and traffic crash involvement in young adulthood. Traffic injury prevention, 9, 508-514.

Blumstein, A., Cohen, J., Roth, J. A., \& Visher, C. A. (1986). Criminal Careers and "Career Criminals". Vol. 1 and Vol. II. Washington, DC: National Academy Press.

Breuvart, J., Algan, A., \& Selosse, J. (1974). Que deviennent-t-ils? Étude comparative des niveaux d'intégration sociale d'une population de mineurs de justice. Vaucresson: Centre de formation et de recherche de l'éducation surveillée.

Briggs, J., \& Peat, F. D. (1989). Turbulent mirror. New York: Harper and Row.

Broidy, L. M., Nagin, D. S., Tremblay, R. E., Bates, J. E., Brame, B., Dodge, K., Fergusson, D., Horwood, J., Loeber, R., Laird, R., Lynam, D., Moffitt, T., Pettit, M. B., \& Vitaro, F. (2003). Developmental trajectories of childhood disruptive behaviors and adolescent delinquency: A six site cross-national study. Developmental Psychology, 39, 222-245. 
Cullen, F. T. (1985). Rethinking crime and deviance theory: The emergence of a structural tradition. Totowa: Rowman \& Allanheld.

Dembo, R., Wareham, J., \& Schmeidler, J. (2007). Drug use and delinquent behavior, A growth model of parallel processes among high-risk youths. Criminal justice and behavior, 34, 680-696.

Elder, G. H. (1992). Life course. In E. F. Borgotta \& M. L. Borgotta (Eds.), The encyclopedia of sociology (1120-1130). New York: Macmillan.

Fréchette, M., \& Le Blanc, M. (1987). Délinquances et délinquants. Boucherville: Gaetan Morin.

Gleick, J. (1987). Chaos, making of a new science. New York: Vicking Penguin.

Glueck, S., \& Glueck, E. (1930). Five Hundred Criminal Careers. New York: Knopf.

— (1937). Later Criminal Careers. New York: The Commonwealth Fund.

— (1940). Juvenile Delinquents Grow Up. New York: Commonwealth Fund.

(1943). Criminal Careers in Retrospect. New York: The Commonwealth Fund.

(1968). Delinquents and Non-delinquents in perspective. Cambridge: Harvard University Press.

Gottfredson, M. A., \& Hirschi, T. (1990). A general theory of crime. Stanford: Stanford University Press.

Hirschi, T. (1969). Causes of delinquency. Berkely: University of California Press.

Jessor, R., \& Jessor, S. L. (1977). Problem behavior and psychosocial development. New York: Academic Press.

Kandel, D. (2002). Stages and patbways of drug involvement. Cambridge: Cambridge University Press.

Lacourse, E., Nagin, D. S., Vitaro, F., Côté, S., Arseneault, L., \& Tremblay, R. E. (2006). Prediction of early-onset deviant group affiliation. Archives of general psychiatry, 63, 362-368.

Lanctôt, N., \& Le Blanc, M. (2002). Explaining adolescent females' involvement in general deviance: Towards an integration of theoretical perspectives. Crime and justice, 26, 113-202.

Laub, J. H., Nagin, D. S., \& Sampson, R. J. (1998). Trajectories of change in criminal offending: Good marriages and the desistance process. American journal of Sociology, 63, 225-238.

Le Blanc, M. (1995). Common, temporary, and chronic delinquencies: Prevention strategies during compulsory school. In P-O Wikström, J. McCord \& R. W. Clarke (Eds.), Integrating crime prevention strategies: Motivation and opportunity (169-205). Stockholm: The National Council for Crime Prevention.

(1997). A generic control theory of the criminal phenomenon, the structural and the dynamical statements of an integrative multilayered control theory. Advances in theoretical criminology, 7, 215-286.

(1999). Comportements et adolescents violents, un phénomène spécifique. In J. Proulx, M. Cusson \& M. Ouimet (Eds.), Les violences criminelles (319353). Québec: Presses de l'Université Laval. 
(2002). The offending cycle, escalation and de-escalation in delinquent behavior, A challenge for criminology. International Journal of comparative and applied criminal justice, 26, 53-84.

(2005a). An integrative personal control theory of deviant behavior answers to contemporary empirical and theoretical developmental criminology issues. Advances in Criminological Theory, 14, 125-164.

- (2005b). Les adolescents montréalais et les psychotropes au cours de quatre décennies: développement de la consommation, trajectoires et profils des consommateurs, stratégie et efficacité de l'intervention. In S. Brochu, L. Guyon, \& M. Landry (Eds.), Jeunesse et toxicomanie: bilan des recherches québécoises sur l'usage et l'abus de substances chez les jeunes et leurs familles (143-202). Québec: Presses de l'Université Laval.

(2006). Self-Control and Social Control of Deviant Behavior in Context: Development and Interactions along the Life Course. In P.-O. Wikstrom \& R. Sampson (Eds.), The explanation of crime (195-242). Cambridge: Cambridge University Press.

(2009). The development of deviant behavior, its self-regulation. Monatsschrift fuer Kriminologie und Strafrechtsreform, 91 (4-5), 117-136.

(2010). MASPAQ: Mesures de l'adaptation sociale et psychologique pour les adolescents québécois. Montréal: Université de Montréal.

— (Inédit). Délinquances et délinquants. Montréal: Presses de 1'Université de Montréal.

\& Bouthillier, C. (2003). A developmental test of the general deviance syndrome with adjudicated girls and boys using hierarchical confirmatory factor analysis. Criminal behavior and mental bealth, 13, 81-105.

\& Fréchette, M. (1989). Male Criminal Activity: Its Development from Childhood to Adulthood. New York: Springer-Verlag.

— \& Girard, S. (1998). Psychotropes et délinquance: séquences développementales et enchâssement. Psychotropes, 4, 69-91.

- \& Kaspy, N. (1998). Trajectories of delinquency and problem behavior: Comparison of synchronous and non-synchronous paths on social and personal control characteristics of adolescent. Journal of Quantitative Criminology, 14, 181-214.

— \& Loeber, R. (1998). Developmental criminology upgraded. Crime and Justice 23, 149-232.

— Morizot, J., \& Lanctôt, N. (2002). Adjudicated Males Self-reported Criminality Trajectories From Adolescence to Midlife. American Society of Criminology. Annual Meeting, 13-16 November, Chicago.

Lerner, R. M. (2002). Concepts and theories of human development. New York: Random life. Cambridge: Harvard University Press.

Loeber, R., \& Hay, D. (1997). Key issues in the development of aggression and violence from childhood to early adulthood. Annual Review in Psychology, 48, 371-410.

Loeber, R. \& Le Blanc, M. (1990). Toward a Developmental Criminology. Crime and Justice, $12,375-473$. 
Loeber, R., Smalley, M. M., Keenan, K., \& Zhang, Q. (1997a). A prospective replication of developmental pathways in disruptive and delinquent behavior. In R. B. Cairns (Ed.), The Individual as a Focus in Developmental Research (128-140). Beverly Hills: Sage.

Loeber, R. K., Lacourse, E., \& Homish, D. L. (2006). Homicide, violence and developmental trajectories. In R. E. Tremblay, W. W. Hartup \& J. Archer (Eds.), Developmental origins of aggression (202-222). New York: Guilford Press.

Matza, D. (1957). Delinquency and drift. New York: Wiley.

McDonald, J. M., Haviland, A., \& Morral, A. R. (2009). Assesing the relationship between violent and nonviolent criminal activity among serious adolescent offenders. Journal of research on crime and delinquency, 46, 553-580.

Moffitt, T. E. (1993). Adolescence-limited and life-course persistent antisocial behavior: A developmental taxonomy. Psychological Review, 100, 674-701.

Morizot, J., \& Le Blanc, M. (2007). Behavioral, Self, and Social Control Predictors of the Normative Process of Desistance from Criminal Activity: A Test of the Launch- and Contemporaneous-Effect Models. Journal of Contemporary Criminal Justice, 21, 50-71.

Nagin, D. S., \& Tremblay, R. E. (1999). Trajectories of boys' physical aggression, opposition and hyperactivity on the path to physically violent and nonviolent juvenile delinquency. Child development, 70, 1181-1196.

Osgood, D. W., Johnston, L. D., O’Malley, P. M., \& Bachman, J. G. (1988). The generality of deviance in late adolescence and early adulthood. American Sociological Review, 53, 81-93.

Piquero, A. R., Farrington, D. P., \& Blumstein, A. (2003). The criminal career paradigm. Crime and Justice, 30, 359-506.

Piquero, A. R., Farrington, D. P., \& Blumstein, A. (2007). Key issues in criminal career research. Cambridge: Cambridge University Press.

Sampson, R. J., \& Laub, J. H. (1993). Crime in the making, pathways and turning points through life. Cambridge: Harvard University Press.

Skardhamar, T. (2009). Reconsidering the theory of adolescent-limites and life-course persistent anti-social behaviour. British Journal of Criminology, 49, 863-878.

van Lier, A. C., Vitaro, F., Barker, E. D., Koot, H. M., \& Tremblay, R. E. (2009). Developmental links between trajectories of physical violence, vandalism, theft, and alcoholodrug use from childhood to adolescence. Journal of abnormal child psychology, 37, 481-492

Vitaro, F., Wanner, B., Ladouceur, R., Brendgen, M., \& Tremblay, R. E. (2004). Trajectories of gambling during adolescence. Journal of gambling studies, 20, 47-69.

Wanner, B., Vitaro, F., Carbonneau, R., \& Tremblay, R. E. (2009). Crossedlagged links among gambling, substance use and delinquency from midadolescence to young adulthood: additive and moderating effects of common risk factors. Psychology of addictive behaviors, 23, 91-104. 
ABSTRACT - This paper outlines the developmental paradigm for criminology. This perspective has for object the within individual changes in deviant behavior along the life-course. Deviant behavior is conceptualized and empirically verified as a hierarchical syndrome composed of twelve types and four domains of deviant behaviors. The parameters for the description of the forms of deviant behavior are defined. The mechanisms of the cycle of deviant behavior are specified in terms of activation, aggravation and desistance. This paper proposes that the deviant behavior syndrome auto-regulates itself according to the principles of the chaos-ordre perspective in science and the knowledge of developmental psychology.

KEYWORDS - Deviant behavior, development, quantitative and qualitative changes, mechanisms of activation, aggravation and desistance, auto-regulation.

RESUMEN - Este artículo resume los avances de la criminología en la presentación de los orígenes y las etapas de la construcción del paradigma de desarrollo para la comprensión de la conducta desviante de las personas. Luego de definir los objetos de este paradigma, el artículo describe la estructura jerárquica del síndrome de la conducta desviante. Se precisan los parámetros que permiten describir cada una de las formas de este síndrome en su forma actual y los cambios por los que ha pasado. Se exponen los mecanismos de la construcción (activación, agravación y desistimiento) y las trayectorias de las formas de la conducta desviante. Esta presentación del paradigma de desarrollo se completa con un cuadro de su autorregulación y una indicación de su aplicación al entendimiento del funcionamiento de las causas y las formas de la conducta desviante.

PALABRAS CLAVE - Conducta desviante, desarrollo, cambios cuantitativos y cualitativos, mecanismos de activación, de agravación y de desistimiento, autorregulación. 